Artículo científico

Volumen 31(2):419-431. Mayo-agosto, 2020

e-ISSN 2215-3608, doi:10.15517/am.v31i2.39485

http://www.revistas.ucr.ac.cr/index.php/agromeso

\title{
Mapeo de un gen implicado en segregación distorsionada en poblaciones interespecíficas de tomate ${ }^{1}$
}

\section{Mapping of a gene involved in distorted segregation in interspecific tomato populations}

\author{
Walter Barrantes-Santamaría ${ }^{2}$, Antonio Granell ${ }^{3}$, Antonio J. Monforte ${ }^{3}$
}

1 Recepción: 5 de noviembre, 2019. Aceptación: 9 de marzo, 2020. Este trabajo formó parte de la tesis doctoral en Biotecnología Vegetal del primer autor. Fue financiada por el Consejo Superior de Investigaciones Científica de España y por la Oficina de Asuntos Internacionales y Cooperación Externa de la Universidad de Costa Rica.

2 Universidad de Costa Rica, Estación Experimental Agrícola Fabio Baudrit, Programa de recursos fitogenéticos. Alajuela, Costa Rica. walter. barrantes@ucr.ac.cr (autor para correspondencia; https://orcid.org/0000-0002-5288-451X).

3 Instituto de Biología Molecular y Celular de Plantas (IBMCP), Universitat Politécnica de Valencia (UPV)-Consejo Superior de Investigaciones Científicas (CSIC), Ingeniero Fausto Elio, s/n46022 Valencia, España. agranell@ibmcp.upv.es (https://orcid.org/0000-00034266-9581), amonforte@ibmcp.upv.es (https://orcid.org/0000-0003-3461-3094).

\section{Resumen}

Introducción. La segregación distorsionada (SD) ocurre cuando los genotipos esperados no corresponden a los observados, lo que favorece alelos de un solo parental. Este fenómeno se observó en poblaciones intermedias provenientes del cruce entre Solanum pimpinellifolium y el cultivar Moneymaker de Solanum lycopersicum, desarrolladas durante el proceso de construcción de una genoteca de líneas de introgresión. Objetivo. Obtener recombinantes informativos que permitan mapear físicamente una región con SD asociado a la especie silvestre Solanum pimpinellifolium. Materiales y métodos. La investigación se desarrolló en el Instituto de Biología Molecular y Celular de Plantas (IBMCP) adscrito al Consejo Superior de Investigaciones Científicas (CSIC) con sede en la Universidad Politécnica de Valencia, España. Se cribó una población de 2000 plantas para identificar recombinantes en dicha región, con una modificación de la técnica fusión de alta resolución (high resolution melting, HRM-Multiplex). Estos recombinantes se autofecundaron, y mediante el estadístico Chi-cuadrado se determinó si marcadores SNP identificados dentro de la región diana tenían una segregación normal (1:2:1) o distorsionada para cada recombinante informativo seleccionado. Resultados. Se generaron e identificaron 54 recombinantes informativos, que se agruparon en 10 bins de acuerdo con el sitio físico de recombinación. Se logró acotar la región con segregación distorsionada hasta obtener un tamaño final de $84 \mathrm{~Kb}$, la cual se localizó en el extremo distal del brazo largo del cromosoma 4. Esta región contiene gran cantidad de genes, algunos de los cuales están relacionados con procesos de fecundación, esterilidad y división celular entre otros, que podrían estar relacionados con el fenómeno estudiado. Conclusión. Se localizó un gen que provoca una distorsión en la segregación en un intervalo de 84 Kb y que posiblemente sea el gen Ge descrito por Rick en 1966.

Palabras claves: marcadores genéticos, genotipado masivo, curvas de fusión de alta resolución, recombinación. 


\begin{abstract}
Introduction. Distorted segregation (SD) occurs when the expected genotypes do not correspond to those observed, which favors single parent alleles. This phenomenon was observed in intermediate populations from the cross between Solanum pimpinellifolium and the Moneymaker cultivar of Solanum lycopersicum, developed during the construction process of a library of introgression lines. Objective. Obtain informative recombinants that allow physically mapping a region with SD associated with the wild Solanum pimpinellifolium species. Materials and methods. The research was carried out at the Institute of Molecular and Cellular Biology of Plants (IBMCP) attached to the Higher Council for Scientific Research (CSIC) based at the Universidad Politecnica de Valencia, Spain. A population of 2000 plants was screened to identify recombinants in that region, with a modification of the high-resolution melting technique (HRMMultiplex). These recombinants were self-fertilized, and through the Chi-square statistic it was determined whether SNP markers identified within the target region had a normal (1:2:1) or distorted segregation for each informative recombinant selected. Results. Fifty-four informative recombinants were generated and identified, grouped into 10 bins according to the physical recombination site. It was possible to delimit the region with distorted segregation until obtaining a final size of $84 \mathrm{~Kb}$, which was located at the distal end of the long arm of chromosome 4 . This region contains a large number of genes, some of which are related to fertilization processes, sterility and cell division among others, which could be related to the studied phenomenon. Conclusion. A gene was found, that causes a segregation distortion in an interval of $84 \mathrm{~Kb}$ and possibly is the gene Ge described by Rick in 1966.
\end{abstract}

Keywords: genetic markers, massive genotyping, high resolution fusion curves, recombination.

\title{
Introducción
}

La segregación distorsionada (SD) ocurre cuando la frecuencia de genotipos/alelos observados se desvía significativamente de las frecuencias mendelianas esperadas para una población dada (Koide et al., 2012). Este fenómeno descubierto en plantas de maíz por Mangelsdorf y Jones (1926), se ha descrito en un gran número de especies como tomate (Rick, 1966), arroz (Nakagahra, 1972), trigo (Endo, 1990), cebada (Graner et al., 1991), algodón (Rooney et al., 1991), sorgo (Pereira et al., 1994), alfalfa (Echt et al., 1994), café (Ky et al., 2000), garbanzo (Castro et al., 2011), algodón (Dai et al., 2017), papa (Manrique-Carpintero et al., 2016) y petunia (Guo et al., 2017). Al igual que ha ocurrido para otros caracteres/procesos con base genética, la utilización de marcadores moleculares ha sido una herramienta muy útil en el mapeo de regiones con SD, presente en todo tipo de población recombinante $\left(\mathrm{F}_{2}\right.$, retrocruces, dobles haploides, líneas consanguíneas recombinantes y líneas de introgresión). Investigaciones hechas en sorgo, tomate, maíz, repollo, algodón, entre otros, han demostrado que las desviaciones observadas en la segregación son más acentuadas cuanto más distantes sean las especies cruzadas (Xu et al., 1997; Lu et al., 2002; Li et al., 2007).

Las distorsiones que se observan en la segregación de un carácter o marcador pueden deberse a una multitud de factores que pueden tener origen genético, ambiental o ser producto de la interacción entre ambos (Xu et al., 1997). Las alteraciones que conducen a la SD pueden producirse antes o después del desarrollo del cigoto y, en consecuencia se podrían clasificar en precigóticas o postcigóticas. Antes de la formación del cigoto, la SD estaría ocasionada por alteraciones en las proporciones de los alelos en los gametos y, consecuentemente por cambios en las proporciones genotípicas de cigotos; por ejemplo, alteraciones que resultan en la letalidad o aborto del polen (Rick, 1966), la desigualdad de la capacidad de los tubos polínicos en la polinización (Mangesldorf y Jones, 1926) y la fertilización preferencial (Endo, 1990), el desarrollo meiótico femenino en Mimulus (Findley et al., 2015). El aborto del polen y la competencia de los tubos polínicos son ejemplos de selección de gametos masculinos, y suelen 
ser más frecuentes si se compara con los gametos femeninos (Xu et al., 1997; Song et al., 2006); sin embargo, la SD puede ocurrir en ambos gametos (Rick, 1966). Recientemente, Tovar-Méndez et al. (2014) reportaron que la proteína HT está asociada a los complejos S-RNasa dependiente y S-RNasa independiente, impidiendo condiciones para favorecer cruces entre polen de $S$. lycopersicum y $S$. pennelli, S. habrochaites y S. arcanum.

Al desarrollar poblaciones donde intervienen especies silvestres de tomate, es frecuente que se encuentren regiones genómicas en donde la recombinación se suprime y/o las frecuencias alélicas observadas son diferentes a las esperadas. La frecuencia de estos fenómenos aumenta cuanto más distantes filogenéticamente sean las especies (Anderson et al., 2010). La primera publicación del fenómeno de segregación distorsionada en tomate fue la de Rick (1966), este autor definió que la causa de la SD era un gen al que llamó eliminador (Ge), y propuso que este gen presentaba tres formas alélicas $\left(\mathrm{Ge}^{\mathrm{p}}, \mathrm{Ge}^{\mathrm{c}}\right.$ y $\left.\mathrm{Ge}^{\mathrm{n}}\right)$, y no son viables los gametos que llevan el alelo $\mathrm{Ge}^{\mathrm{c}}$ en plantas con genotipo heterocigota $\left(\mathrm{Ge}^{\mathrm{p}} \mathrm{Ge}^{\mathrm{c}}\right)$. El alelo $\mathrm{Ge}^{\mathrm{p}}$ (predominante en S. pimpinellifolium) es seleccionado preferentemente sobre $\mathrm{Ge}^{\mathrm{c}}$ y $\mathrm{Ge}^{\mathrm{n}}$ (predominante en $S$. lycopersicum), y ocurre por igual en los gametos masculinos y femeninos, con una penetración cercana al $95 \%$ cuando se combinan los efectos en ambos gametos (Rick, 1970). En las últimas décadas, muchos autores han observado SD trabajando con poblaciones de tomate que implicaban diferentes especies silvestres relacionadas (Pelham, 1968; Zamir y Tadmor, 1986; deVincente y Tanksley, 1993; Bernacchi y Tanksley, 1997; Chetelat y Meglic, 2000).

Durante el desarrollo de una genoteca de líneas de introgresión (IL) entre la especie silvestre S. pimpinellifolium y el cultivar Moneymaker de S. lycopersicum, se presentó un patrón de segregación en marcadores SNPs localizados en el cromosoma 4 que favorecieron los alelos de $S$. pimpinellifolium sobre los de $S$. lycopersicum, lo que hizo prácticamente imposible eliminar una introgresión silvestre no deseada de las plántulas. Como consecuencia de ello, un fragmento homocigoto de S. pimpinellifolium de dicha región está presente en más del $50 \%$ de la IL desarrolladas (Barrantes et al., 2014). Esta situación motivó estudiar este fenómeno con más detalle, para lo cual se planteó como objetivo obtener recombinantes informativos que permitan mapear físicamente la región con SD asociada a la especie silvestre Solanum pimpinellifolium.

\section{Materiales y métodos}

La investigación se desarrolló durante los años 2010 a 2014 en el Instituto de Biología Molecular y Celular de Plantas adscrito al Consejo Superior de Investigaciones Científicas (CSIC) con sede en la Universidad Politécnica de Valencia, España.

\section{Localización inicial de la región con SD}

La definición inicial de la región cromosómica que presentó segregación distorsionada se basó en la información obtenida del genotipado masivo realizado a poblaciones intermedias de retocruce avanzado (BC2, BC3), provenientes del cruce entre S. lycopersicum y $S$. pimpinellifolium (acc. TO-937), que presentaron segregación no correspondiente a las esperadas y que formaron parte en la construcción de una genoteca de líneas de introgresión (Barrantes et al., 2014).

Adicionalmente, y con el objetivo de corroborar la localización y tamaño inicial aproximado de la región con $\mathrm{SD}$, se usaron los resultados de un genotipado masivo, realizado mediante el chip de SOLCAP (Slim et al., 2012), a una población de líneas recombinantes consanguíneas (RILs), desarrollada por Fernández-del-Carmen et al. (2011), a partir de los mismos parentales usados en esta investigación. 


\section{Población vegetal y generación inicial de recombinantes}

El material vegetal inicial utilizado fueron plantas pertenecientes a una población del retrocruce avanzado $\left(\mathrm{BC}_{3} \mathrm{~S}_{1}\right)$, derivada del cruce entre $S$. lycopersicum cv Moneymaker (MM) y S. pimpinellifolium (acc. TO-937). Dichas plantas presentaron segregaciones en marcadores SNPs distintas a las esperadas para un evento de autofecundación si se considera un solo locus. De esta población original se seleccionaron, por medio de la técnica de fusión de alta resolución (HRM, por sus siglas en inglés), genotipos heterocigotos para la región cromosómica que presentaba el fenómeno de segregación distorsionada (SD). Las plantas generaron recombinantes por medio de autofecundaciones sucesivas hasta lograr una población final de mapeo de 2000 plantas, distribuidas en un ámbito de hasta seis generaciones de autofecundación $\left(\mathrm{BC}_{3} \mathrm{~S}_{1}\right.$ hasta $\left.\mathrm{BC}_{3} \mathrm{~S}_{6}\right)$.

\section{Extracción de ADN}

El ADN genómico se aisló de hojas primarias jóvenes en plántulas de almácigo, con base en el método de Doyle y Doyle (1990), con ligeras modificaciones. Se recolectaron $100 \mathrm{mg}$ de tejido fresco de cada genotipo y se depositaron en microtubos de plástico de $1,5 \mathrm{ml}$, a los cuales se les agregó dos balines de acero de $3 \mathrm{~mm}$ y $370 \mathrm{ml}$ de buffer de extracción. Se trituraron las muestras en un Geno/Grinder 2000 (SPEX Sample Prep, Nueva Jersey, USA) durante 5 min a $1400 \mathrm{rpm}$. El ADN extraído se diluyó en agua Milli-Q hasta lograr una concentración final de 10 a $20 \mathrm{ng} \mathrm{ml}^{-1}$.

\section{Búsqueda de recombinantes por medio de HRM}

La búsqueda de recombinantes para el mapeo genético de la región con $\mathrm{SD}$, se realizó utilizando una adaptación de la técnica de genotipado con marcadores SNP de fusión de alta resolución (HRM) (Simko, 2016). En una primera etapa se cribaron las 2000 plantas con marcadores SNPs con base en la técnica HRM-múltiplex, que consiste en una PCR tiempo real con el empleo de una combinación de dos marcadores que flanquean la región previamente definida con SD (3,16 Mb). Se usaron los marcadores: aguas arriba (secuencias de ADN localizadas hacia el lado proximal del cromosoma), Solcap_snp sl _ 47229F CAAGCCACCAGATTCCTTCC y Solcap_snp sl 47299R TGGAATACGACTTACTCAGCGAGA, y aguas abajo (secuencias de ADN localizadas hacia el lado distal del cromosoma), Solcap _snp_sl_ 4164F CGGAGAGTAGTTGGAGGAACACC y Solcap_snp sl 4164R GAGAAGCTGGAGAAGGTTGGG. En una segunda etapa se generaron curvas de HRM simples con marcadores SNP localizados en el intervalo del mapa previamente definido (Cuadro 1), los cuales se evaluaron en los recombinantes encontrados en la primera etapa que presentaron una o ambas regiones flanqueantes heterocigotas. Las condiciones de PCR-HRM fueron: volumen total de mezcla de $10 \mu \mathrm{l}, 5 \mu \mathrm{l}$ de master mix (Kit de PCR HRM Type-it, QIAGEN, Hilden, Alemania), $0.175 \mu \mathrm{l}$ (para PCR-HRM multiplex) y 0,35 ul (para PCR-HRM simple) de cada uno de los oligos $(10 \mu \mathrm{M}), 2 \mu \mathrm{l}$ de ADN genómico y 2,3 $\mu \mathrm{l}$ de agua libre de ARNasa. Las reacciones se establecieron en un sistema de PCR en tiempo real Rotor-Gene Q (QIAGEN, Hilden, Alemania).

La PCR-HRM se corrió bajo las siguientes condiciones: una etapa de preamplificación a $95{ }^{\circ} \mathrm{C}$ durante 10 min, seguido de cuarenta ciclos con una primera etapa a $95{ }^{\circ} \mathrm{C}$ por $10 \mathrm{~s}$ y una segunda etapa a $58{ }^{\circ} \mathrm{C}$ por 30 s. El análisis HRM se realizó inmediatamente después de completar la amplificación por PCR. El intervalo de temperatura evaluado fue de 65 a $85^{\circ} \mathrm{C}$, con incrementos de $0,1{ }^{\circ} \mathrm{C}$ y un tiempo de retención de $2 \mathrm{~s}$ para cada paso de adquisición. Se usó el software Rotor Gene Q (QIAGEN, Hilden, Alemania), para definir las condiciones de PCR, monitorear la amplificación en tiempo real y para ver y analizar las curvas de fusión. 
Cuadro 1. Secuencias de los marcadores SNP polimórficos utilizados en el genotipado de los 54 recombinantes informativos. IBMCP/ UPV, Valencia-España. 2013.

Table 1. Sequences of the polymorphic SNP markers used in the genotyping of the 54 informative recombinants. IBMCP/UPV, Valencia-España. 2013.

\begin{tabular}{|c|c|c|c|}
\hline Número & Nombre marcador & Secuencia $(\mathbf{F} / \mathbf{R})$ & Mapa \\
\hline 1 & Solcap_snp_sl_47229 F & CAATGAAATTAGGCAGGTGGC & MAPA SOLCAP \\
\hline 2 & Solcap_snp_sl_47229 R & TGCAAGTCCTAGCCTGGCC & MAPA SOLCAP \\
\hline 3 & Solcap_snp_sl_47397 F & CAAATTGAACTCACAGTATCGGTTG & MAPA SOLCAP \\
\hline 4 & Solcap_snp_sl_47397 R & TGAGTAAGTCGTATTCCACTATATT & MAPA SOLCAP \\
\hline 5 & Solcap_snp_sl_47413 F & TGCTAAGTCATGGACACCAACG & MAPA SOLCAP \\
\hline 6 & Solcap_snp_sl_47413 R & САСССТTTTCATTGGAGTCCC & MAPA SOLCAP \\
\hline 7 & Solcap_snp_sl_47457 F & TCCTCCCTTCGAGCTGCA & MAPA SOLCAP \\
\hline 8 & Solcap_snp_sl_47457 R & GTAGAGCCACGACATCATCCAG & MAPA SOLCAP \\
\hline 9 & Solcap_snp_sl_47656 F & TGTGAAACCCTAACTGGATTTCG & MAPA SOLCAP \\
\hline 10 & Solcap_snp_sl_47656 R & CCTTAACATACGCACTCTCCGTT & MAPA SOLCAP \\
\hline 11 & Solcap_snp_sl_3827 F & GCATTGAAGCCCAGAGGAAC & MAPA SOLCAP \\
\hline 12 & Solcap_snp_sl_3827 R & ATTCTAAAAGCTCATCCTCGCCTA & MAPA SOLCAP \\
\hline 13 & Solcap_snp_sl_47694 F & TTGTTCGTCTCCGTCAAATGC & MAPA SOLCAP \\
\hline 14 & Solcap_snp_sl_47694 R & GGGTACGCGACAACGAGC & MAPA SOLCAP \\
\hline 15 & Solcap_snp_sl_47697 F & TGGCGAGCACTAAATCCCA & MAPA SOLCAP \\
\hline 16 & Solcap_snp_sl_47697 R & TCСТCССТTCGAGCTGCA & MAPA SOLCAP \\
\hline 17 & Solcap_snp_sl_3920F & GCCATCAACGATCAACAACTCTAT & MAPA SOLCAP \\
\hline 18 & Solcap_snp_sl_3920R & ACGTGGTGGTTGAAGATGCA & MAPA SOLCAP \\
\hline 19 & Solcap_snp_sl_47766F & GCAAААCСТTCАТCСССААA & MAPA SOLCAP \\
\hline 20 & Solcap_snp_sl_47766R & GCCACTGCTAAATATGCCCAAT & MAPA SOLCAP \\
\hline 21 & Solcap_snp_sl_4003F & TCAAACAGGGTGGTGCTCTG & MAPA SOLCAP \\
\hline 22 & Solcap_snp_sl_4003R & АATCACTCGAAAAAAAATCATCCAA & MAPA SOLCAP \\
\hline 23 & Solcap_snp_sl_23589F & САСАСТААССТTТGGCAАTCCС & MAPA SOLCAP \\
\hline 24 & Solcap_snp_sl_23589R & TTCAAGGCAGCTCAGGCG & MAPA SOLCAP \\
\hline 25 & Solcap_snp_sl_4164F & CGGAGAGTAGTTGGAGGAACACC & MAPA SOLCAP \\
\hline 26 & Solcap_snp_sl_4164R & GAGAAGCTGGAGAAGGTTGGG & MAPA SOLCAP \\
\hline 27 & Solcap_snp_sl_47742F & CACTCCAAAACATCGTCAGCTC & MAPA SOLCAP \\
\hline 28 & Solcap_snp_sl_47742R & ACTGTTGCATTGCTGTTGGC & MAPA SOLCAP \\
\hline 29 & Solcap_snp_sl_3952F & TCTTTCTTCGTGCAACCTTCTG & MAPA SOLCAP \\
\hline 30 & Solcap_snp_sl_3952R & CGATATAAAGTTGAAGGAGATCGGT & MAPA SOLCAP \\
\hline 31 & Solcap_snp_sl_47749F & TTCTGGGTGCTGTCGGATG & MAPA SOLCAP \\
\hline 32 & Solcap_snp_sl_47749R & TGGCAAGGCCACTCATGA & MAPA SOLCAP \\
\hline 33 & Solcap_snp_sl_3975F & TTTTGATGTTTTCCGTCAATGG & MAPA SOLCAP \\
\hline 34 & Solcap_snp_sl_3975R & GATGCACGAAACAATGTACGAGA & MAPA SOLCAP \\
\hline
\end{tabular}

\section{Mapeo físico de gen (s) implicado (s) en la SD}

En el proceso final de mapeo, los recombinantes informativos se autopolinizaron para generar poblaciones F2. Los que lograron producir semilla en cantidad suficiente (al menos cincuenta semillas F2) se plantaron para realizar posteriormente las pruebas de progenie. A cada miembro de estas poblaciones F2 se le extrajo ADN genómico y se cribaron con un marcador SNP localizado dentro de la región heterocigota y se determinó el genotipo. Se utilizó 
el estadístico Chi-cuadrado $\left(\mathrm{X}^{2}\right)$ para determinar si la segregación del marcador era normal (segregación esperada $1: 2: 1)$ o distorsionada.

\section{Resultados}

\section{Detección de la segregación distorsionada en la población inicial}

Algunas familias de las poblaciones intermedias presentaron a la altura del cromosoma 4 segregaciones con genotipos que favorecieron los alelos del parental silvestre $S$. pimpinellifolium sobre los alelos del cultivar comercial S. lycopersicum, evidenciando segregación distorsionada (Cuadro 2).

Cuadro 2. Evidencia inicial del fenómeno de segregación distorsionada en poblaciones de retrocruce avanzado. Segregaciones esperadas/observadas y resultado de la prueba de Chi-cuadrado. Realizada a distintas poblaciones desarrolladas a partir del cruce entre S. lycopersicum variedad MM y S. pimpinellifolium acc.TO-937. IBMCP/UPV, Valencia-España. 2010-2014.

Table 2. Initial evidence of the distorted segregation phenomenon in advanced backcross populations. Expected/observed segregations and Chi-square test result. Carried out to different populations developed from the S. lycopersicum variety MM and S. pimpinellifolium acc.TO-937 cross. IBMCP/UPV, Valencia-España. 2010-2014.

\begin{tabular}{ccccccccc}
\hline Población & \multicolumn{7}{c}{ Segregación (genotipos) esperados (\%) } & \multicolumn{2}{c}{ Segregación (genotipos) observados (\%) } & $\mathbf{X}^{2}$ & Resultado \\
\hline & $\boldsymbol{l l}$ & $\boldsymbol{l} \boldsymbol{p}$ & $\boldsymbol{p} \boldsymbol{p}$ & $\boldsymbol{l l}$ & $\boldsymbol{l} \boldsymbol{p}$ & $\boldsymbol{P} \boldsymbol{p}$ & & \\
$\mathrm{BC}_{2}$ & 75 & 25 & 0 & 33 & 66 & 0 & $64^{* *}$ & $\mathrm{SD}$ \\
$\mathrm{BC}_{3}$ & 50 & 50 & 0 & 40 & 60 & 0 & $5^{*}$ & $\mathrm{SD}$ \\
$\mathrm{BC}_{3} \mathrm{~S}_{1}$ & 25 & 50 & 25 & 6 & 31 & 63 & $165^{* *}$ & $\mathrm{SD}$ \\
\hline
\end{tabular}

Los porcentajes corresponden al genotipado con HRM con marcadores SNP localizados en la región distal del brazo largo del cromosoma 4. La letra $\boldsymbol{l l}$ genotipo homocigoto $S$. lycopersicum, $\boldsymbol{l p}$ genotipo heterocigoto y $\boldsymbol{p} \boldsymbol{p}$ genotipo homocigoto $S$. pimpinellifolium ac TO-937, SD: segregación distorsionada (*p<0,05, **p<0,0001) / The percentages correspond to HRM genotyping with SNP markers located at the distal region of the long arm of chromosome 4. Letter $\boldsymbol{l l}$ S. lycopersicum homozygous genotype, $\boldsymbol{l} p$ heterozygous genotype, and $p \boldsymbol{p}$ S. pimpinellifolium homozygous genotype ac TO-937, SD: distorted segregation $\left({ }^{*} \mathrm{p}<0.05,{ }^{* *} \mathrm{p}<0.0001\right)$.

\section{Generación e identificación de recombinantes informativos usados en el mapeo}

Todas las combinaciones posibles de curvas derivadas de la fusión, utilizando la técnica PCR-HRM-multiplex se muestran en la Figura 1. Las plantas seleccionadas fueron las que mostraron en la región diana extremos con genotipo heterocigoto $(\boldsymbol{l} \boldsymbol{p})$, o un extremo heterocigoto y el otro extremo un genotipo homocigoto silvestre o comercial $(\boldsymbol{p} p / l l))$. La combinación de genotipos de las Figuras 1b, 1c y 1d, para el cribado de las plantas, consideró además de la forma de la curva de fusión, la temperatura de esta (tm).

Se detectaron 54 recombinantes informativos en la región diana, obtenidos por medio de la técnica PCR-HRM multiplex con un tamaño inicia de 3,16 Mb (Cuadro 3a y 3b) Se agruparon en 10 bins (que consideró el sitio donde ocurrió la recombinación). De este total de recombinantes informativos solo 16 produjeron semilla en cantidades adecuadas para realizar las pruebas de progenie que indicarían si presentaban SD. Algunos recombinantes se ubicaron en el mismo bin (mismo sitio de recombinación, por tanto, se consideraron homólogos), de tal manera que, de los 54 recombinantes informativos obtenidos, solo cinco (recombinantes aguas arriba 4, 13 y 26 y aguas abajo 21 y 54, Cuadro 3a y 3b), se usaron en las pruebas de progenie y en el mapeo final de la región con SD. 

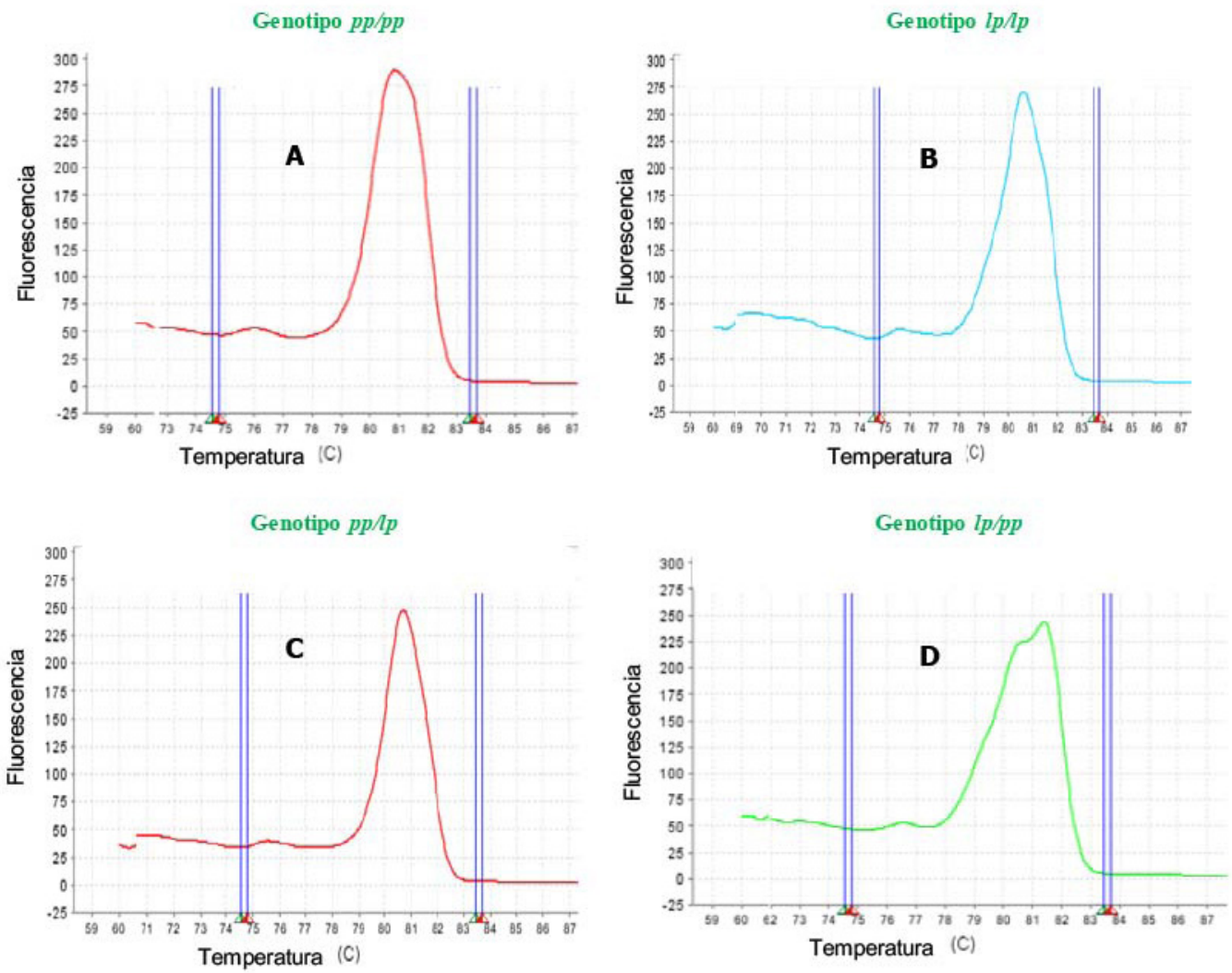

Figura 1. Patrones de las curvas de fusión de PCR-HRM-Multiplex, usadas para identificar recombinantes en una región con segregación distorsionada del cromosoma 4 en tomate. IBMCP/UPV. Valencia-España. 2014. A- genotipo pp/pp: ambos extremos de la región amplificada con genotipo homocigotos $S$. pimpinellifolium; B- genotipo lp/lp: ambos extremos de la región amplificada con genotipo heterocigotos; C- genotipo pp/lp: un extremo la región amplificada con genotipo homocigotos S. pimpinellifolium y el otro extremo heterocigoto y D- genotipo lp/pp: un extremo la región amplificada con genotipo heterocigoto y el otro extremo con genotipo homocigoto S. pimpinellifolium. Mezcla de marcadores SNP usados: aguas arriba Solcap_snp_sl_47229 y aguas abajo Solcap_snp_ sl_4164.

Figure 1. Patterns of the PCR-HRM-Multiplex fusion curves, used to identify recombinants in a region with distorted segregation of chromosome 4 in tomato. IBMCP/UPV. Valencia-España. 2014. A- pp/pp genotype: both ends of the amplified region with $S$. pimpinellifolium homozygous genotype; B- $l p / l p$ genotype: both ends of the region amplified with heterozygous genotype; C- $p p / l p$ genotype: one end the region amplified with $S$. pimpinellifolium homozygous genotype and the other heterozygous end, and $\mathbf{D}$ - $\mathbf{l p} / \mathbf{p p}$ genotype: one end the region amplified with heterozygous genotype and the other one with S. pimpinellifolium homozygous genotype. Mixture of SNP markers used: upstream Solcap_snp_s1_47229 and downstream Solcap_snp_sl_4164.

\section{Tamaño físico final de la región con segregación distorsionada}

Al genotipar los 54 recombinantes informativos obtenidos, se observó (Cuadro 3a y 3b) que a medida que se evaluó con marcadores cercanos a la región distal del brazo largo del cromosoma 4, los alelos de S. lycopersicum (l) 


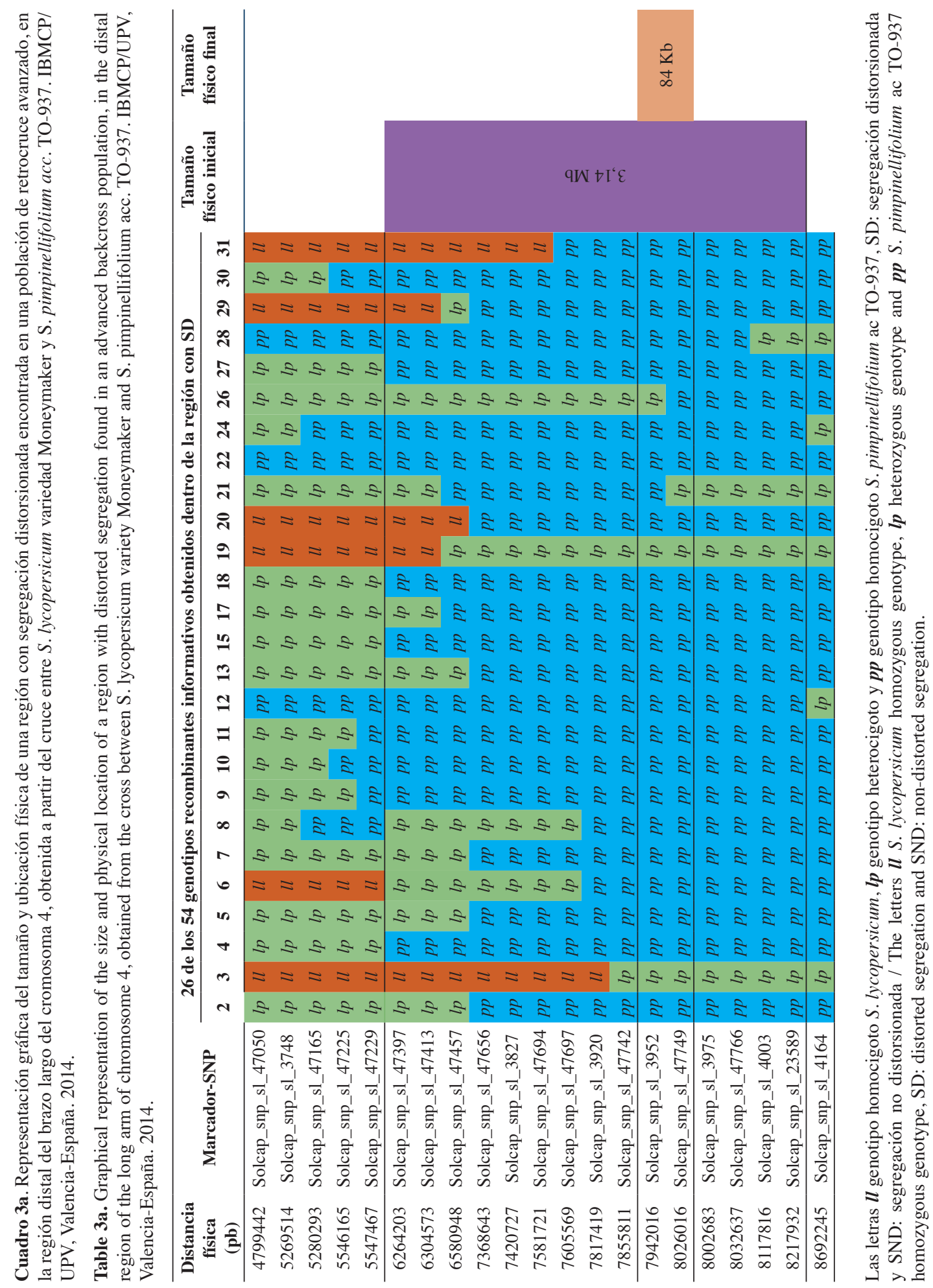




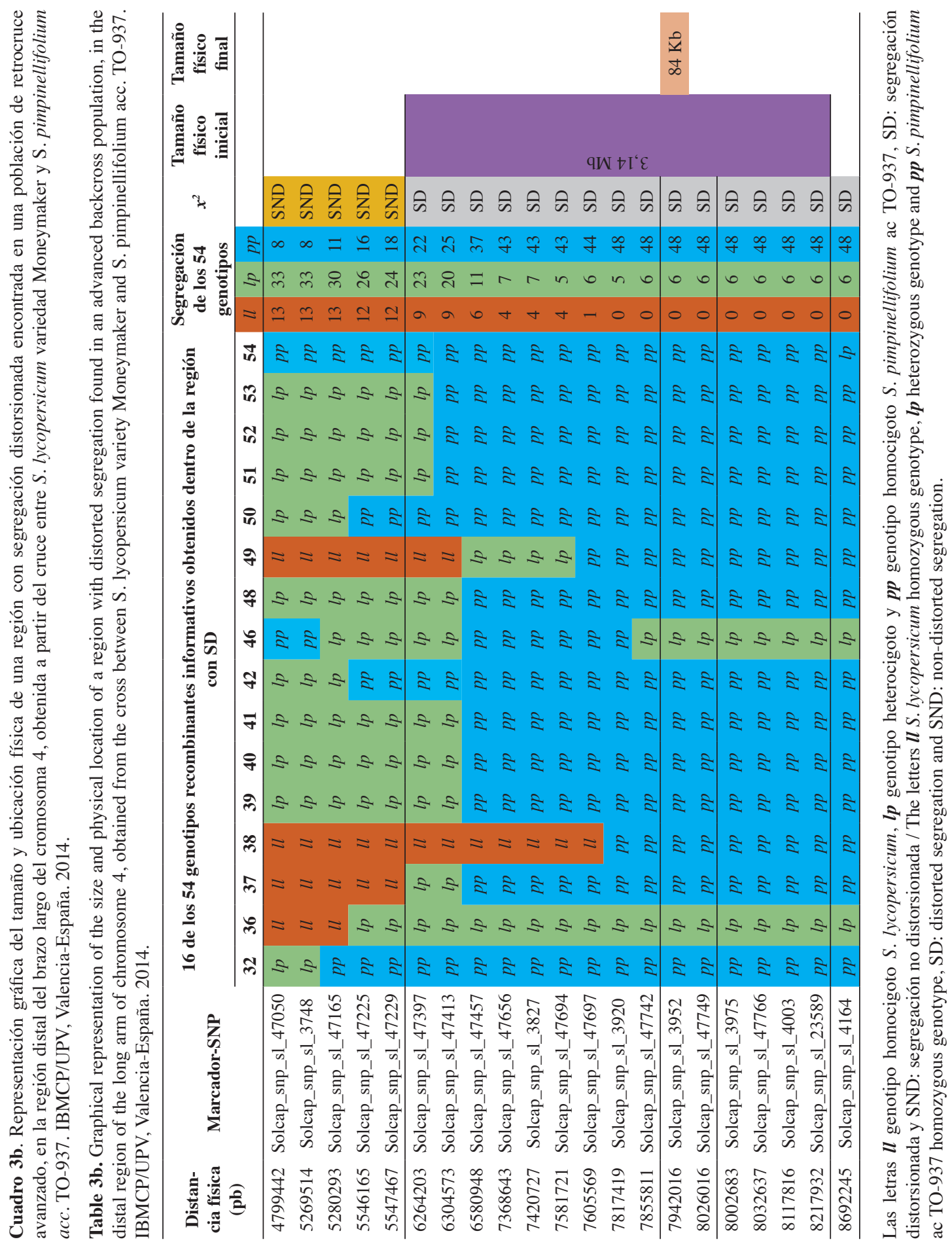


aparecieron cada vez con menor frecuencia, hasta el punto de desaparecer por completo los genotipos homocigotos de $S$. lycopersicum (ll); por el contrario, los alelos silvestres de S. pimpinellifolium ( $\boldsymbol{p}$ ) aparecieron con mayor frecuencia a medida que la ubicación física de los marcadores se aproximaba a la región distal del brazo largo del cromosoma 4; en consecuencia, en la región con máxima distorsión, se encontraron en baja frecuencia genotipos heterocigotos $(\boldsymbol{l} \boldsymbol{p})$ y en alta frecuencia genotipos homocigotos del parental silvestre ( $\boldsymbol{p} \boldsymbol{p})$ y ausentes del todo los genotipos homocigotos de S. lycopersicum (ll).

Con esta información se mejoró la delimitación física de la región estudiada. A partir de los resultados del estadístico Chi-cuadrado realizado a las progenies, los segregantes informativos más valiosos fueron el 4, 13, 21, 26 y 54. La precisión de la ubicación y el tamaño de esta región se mejoró ubicándose con un tamaño final de 84 Mb, localizada según el mapa de Solcap entre los marcadores Solcap_snp_sl_3952 y Solcap_snp_sl_47749.

Según la información presentada en la página del SOL Genomics Network (2014), la región mapeada con SD (84 kb) contiene gran cantidad de genes, algunos de los cuales están relacionados con procesos de fecundación, esterilidad y división celular, que podrían estar relacionados con el fenómeno estudiado.

\section{Discusión}

La cercanía filogenética entre las especies $S$. lycopersicum y $S$. pimpinellifolium presupondría la no existencia de barreras a la compatibilidad entre ambas especies, y aunque esto se cumple, no todas las regiones del genoma se transfieren a la descendencia con la misma eficiencia. Más concretamente se pudo definir que en una región con SD del cromosoma 4, los alelos de la especie silvestre se transmitieron preferentemente frente a los equivalentes del tomate cultivado. Ello provocó supresiones en los eventos de recombinación, que llegaron a ser de cero en algunas secciones de cromosoma dentro de la región con SD. Resultados similares fueron mencionados por Chetelat y Meglic (2000), quienes observaron la presencia de supresiones en la recombinación, en cruces interespecíficos de tomate, siendo más común en poblaciones de retrocruces avanzados, presentándose más en gametos masculinos que en gametos femeninos.

Con la evidencia recopilada en las diferentes pruebas realizadas para estudiar la SD, fue posible hacer una primera descripción de las características que modelan la SD en la región distal del brazo largo del cromosoma 4: 1) la distorsión favoreció los alelos del parental silvestre $(\boldsymbol{p}) ; 2)$ es un fenómeno de tipo precigótico dado que plantas que contienen la región con SD produjeron polen viable (la autofecundación en plantas con SD fue factible) y semillas fértiles con porcentajes de germinación normales en plantas con SD (datos no presentados en este trabajo); 3) afectó tanto a gametos masculinos como femeninos, se observó en las descendencias de las autofecundaciones, y si solo uno de los alelos $\boldsymbol{l}$ fallara, sea macho o hembra, la descendencia debería de ser $50 \%$ de heterocigotos (lp ) y $50 \%$ de homocigotos (pp); sin embargo, la segregación observada fue prácticamente un 0:1:3 (ll:lp:pp), por consiguiente, ambos alelos estuvieron afectados pero no en su totalidad, lo que conllevó la cuarta característica; 4) la penetración no fue del $100 \%$, la mejor evidencia fue la presencia de genotipos heterocigotos $(\boldsymbol{l p})$ en la región con SD. Estas características de la SD guardan mucha similitud con las descritas por Rick (1966; 1970), este autor atribuyó la SD a la presencia de un gen eliminador de gametos; sin embargo, a pesar de que Rick localizó el gen en el cromosoma 4, la posición que él mencionó difiere de la encontrada en el presente estudio, lo cual se podría atribuir a las diferencias entre los mapas usados en cada experimento y, posiblemente recursos utilizados mejoraron sustancialmente la precisión de los resultados.

Las principales consecuencias negativas de enfrentarse a una región con segregación distorsionada, cuando se están desarrollando poblaciones de mejora o de mapeo son la ralentización del proceso de construcción de las poblaciones, un producto final que contiene una introgresión no-diana en un porcentaje considerable de individuos y la posible interferencia (efecto epistástico) de la región con SD en el mapeo de QTLs, que induzca 
a sobreestimar/subestimar el efecto de algún QTLs. Una posible aplicación en mejora genética es hacer selección asistida introgresando un gen (s) de alto valor agronómico en la región con SD en poblaciones pre-mejoradas, ya que, debido al ligamiento que se tendría entre el gen introgresado y la región con segregación distorsionada, la probabilidad que el gen diana de alto valor agronómico y la región con SD co-segregen en poblaciones de mejora es del $100 \%$.

En este caso en particular, dado el gran tamaño físico de la región con SD $(84 \mathrm{~Kb})$, la identificación de genes relevantes asociados al fenómeno aún fue muy preliminar; sin embargo, se observó que en la región con SD hay genes interesantes. Queda por saber si las versiones de estos genes en el genoma de TO-937 presentan alguna diferencia sustancial capaz de explicar por qué en un ambiente heterocigoto los alelos silvestres de esta accesión privan sobre los alelos comerciales. Para dilucidar ello, es necesario acotar aún más la región con SD, a través del desarrollo de más recombinantes que deberán ser interrogados con nuevos marcadores SNP's desarrollados a lo largo de la región estudiada, de tal forma que se pueda ligar uno o más genes al fenómeno del SD identificado en esta investigación.

\section{Conclusiones}

A pesar de la cercanía filogenética que presentan las especies S. lycopersicum y S. pimpinellifolium, existen regiones cromosómicas con diferencias de apareamiento, dando como resultado segregaciones anormales, con proporciones en los genotipos distintas a las esperadas; esto da origen a segregaciones distorsionadas en loci donde la recombinación es casi nula, hasta el punto de llegar a prevalecer un solo genotipo en distintas poblaciones. En esta investigación los alelos silvestres prevalecieron un $100 \%$ sobre los alelos comerciales y esta prevalencia fue del $100 \%$ en la región de mayor distorsión. Esta distorsión en la segregación se ha localizado en un intervalo de 84 $\mathrm{Kb}$ en el brazo largo del cromosoma 4 y, posiblemente corresponde al gen Ge descrito por Rick en 1966.

\section{Literatura citada}

Anderson, L.K., P.A. Covey, L.R. Larsen, P.A. Bedinger, and S.M. Stack. 2010. Structural differences in chromosomes distinguishes species in the tomato clade. J. Cytogen Genome Res. 129:24-34. doi:10.1159/000313850

Barrantes, W., A. Fernández-del-Carmen, G. López-Casado, M.A. González-Sánchez, R. Fernández-Muñoz, A. Granell, and A.J. Monforte. 2014. Highly efficient genomics-assisted development of a library of introgression lines of Solanum pimpinellifolium. Mol. Breed. 34:1817-1831. doi:10.1007/s11032- 014-0141-0

Bernacchi, D., and S.D. Tanksley. 1997. An interspecific backcross of Lycopersicon esculentum x L. hirsutum: linkage analysis and QTL study of sexual compatibility factors and floral traits. Genetics 147:861-877.

Castro, P., J. Rubio, A. Cabrera, T. Millan, and J. Gil. 2011. A segregation distortion locus located on linkage group 4 of the ckickpea genetic map. Euphytica 179:515-523. doi:10.1007/s10681-011-0356-7

Chetelat, R.T., and V. Meglic. 2000. Molecular mapping of chromosome segments introgressed from Solanum lycopersicoides into cultivated tomato (Lycopersicum esculentum). Theor. Appl. Genet. 100:232-241. doi:10.1007/s001220050031

Dai, B., H. Guo, C. Huang, M.M. Ahmed, and Z. Lin 2017. Identification and characterization of segregation distortion loci on cotton chromosome 18. Front. Plant Sci. 7:2037. doi:10.3389/fpls.2016.02037

deVincente, and S.D. Tanksley. 1993. QTL analysis of transgressive segregation in an interspecific tomato cross. Genetics 134:585-596. 
Doyle, J.J., and J.L. Doyle. 1990. Isolation of plant DNA from fresh tissue. Focus 12:13-15.

Echt, C.S., K.K Kidwell, S.J. Knaap, T.C Osborn, and T.J. Mccoy. 1994. Linkage mapping in diploid alfalfa (Medicago sativa). Genome 37:61-71. doi:10.1139/g94-008

Endo, T.R. 1990. Gametocidal chromosomes and their induction of chromosome mutations in wheat. Japan. J. Genet. 65:135152. doi:10.1266/jjg.65.135

Fernández-del-Carmen, G., J. Abad, A. Fernández-Muñoz, A. Granell, and A.J. Monforte. 2011. Applications of the SolCap Illumina SNP array in tomato genetics. Presented at: $8^{\text {th }}$ Solanaceae and $2^{\text {th }}$ Cucurbit Genomics Initiative. $178^{\text {th }}$ Committee on Plant Molecular Design, University-Industry Research Cooperation Societally Applied Scientific Linkage and Collaboration of Japan Society for the Promotion of Sciene, 28 nov- 2 Dic 2011. Kobe, JPN.

Findley, R.F., Y. Dong, A. Saunders, and L. Fishman. 2015. Duplication and adaptive evolution of a key centromeric protein in Mimulus, a genus with female meiotic drive. Mol. Biol. Evol. 32:2694-2706. doi:10.1093/molbev/msv145

Graner, A., A. Jahoor, J. Schondelmaier, H. Siedler, K. Pillen, G. Fischbeck, G. Wenzel, and R.G. Herrmann. 1991. Construction of an RFLP map of barley. Theor. App. Genet. 83:250-256. doi:10.1007/BF00226259

Guo, Y., W.K. Lin, Q. Chen, V.A. Vallejo, and R.M. Warner. 2017. Genetic determinants of crop timing and quality traits in two interspecific Petunia recombinant inbred line populations. Sci. Rep. 7:3200. doi:10.1038/s41598-017-03528-9

Koide, Y., Y. Shinya, M. Ikenaga, N. Sawamura, K. Matsubara, K. Onishi, A. Kanazawa, and Y. Sano. 2012. Complex genetic nature of sex-independent transmission ratio distortion in Asian rice species: the involvement of unlinked modifiers and sex-specific mechanisms. Heredity 108:242-247. doi:10.1038/hdy.2011.64

Ky, C.L., P. Barre, M. Lorieux, P. Trouslot, S. Akaffou, J. Louarn, A. Charrier, S. Hamon, and M. Noirot. 2000. Interspecific genetic linkage map, segregation distortion and genetic conversion in coffee (Coffea sp.) Theor. Appl. Genet. 101:669676. doi: $10.1007 / \mathrm{s} 001220051529$

Li, W., Z. Lin, and X. Zhang. 2007. A novel segregation distortion in intraspecific population of Asian cotton (Gossypium arboretum L.) detected by molecular markers. J. Genet. Genomics 34:634-640. doi:10.1016/S1673-8527(07)60072-1

Lu, H., S.J. Romero, and R. Bernardo. 2002. Chromosomal regions associated with segregation distortion in maize. Theor. Appl. Genet. 105:622-628. doi:10.1007/s00122-002-0970-9

Mangelsdorf, P.C., and D.F. Jones. 1926. The expression of mendelian factors in the gametophyte of maize. Genetics 11:423-455.

Manrique-Carpintero, N.C., J.J. Coombs, R.E. Veilleux, R. Buell, and D.S. Douches. 2016. Comparative analysis of regions with distorted segregation in three diploid populations of potato. G3: Genes, Genomes, Genetics 6:2617-2628. doi:10.1534/ g3.116.030031

Nakagahra, M. 1972. Genetic mechanism on the distorter segregation of marker genes belonging to the eleventh linkage group in cultivated rice. Japan. J. Breed. 22:232-238. doi:10.1270/jsbbs 1951.22.232

Pelham, J. 1968. Disturbed segregation of genes on chromosome 9: gamete promoter., Gp., a new gene. Rep. Tomato Genet. Coop. 18:27-29.

Pereira, M.G., M. Lee, P. Bramel-Cox, J.W. Woodman, J. Doeblen, and R. Whitkus. 1994. Construction of an RFLP map in sorghum and comparative mapping in maize. Genome 37:236-243. doi:10.1139/g94-033

Rick, C.M. 1966. Abortion of male and female gametes in the tomato determined by allelic interaction. Genetics 53:85-96.

Rick, C.M. 1970. The Tomato Ge locus linkage relations and geographic distribution of alleles. Genetics 67:75-85.

Rooney, W.L., and D.M. Stelly. 1991. Preferential transmission and somatic elimination of a Gossypium sturtianum chromosome in G. hirsutum. J. Heredity 82:151-155. doi:10.1093/oxfordjournals.jhered.a111050 
Simko, I. 2016. High-resolution DNA melting analysis in plant research. Trends Plant Sci. 21:528-537. doi:10.1016/j. tplants.2016.01.004

Slim, S.C., G. Durstewitz, J. Plieske, R. Wieseke, M.W. Ganal, A. Van-Deynze, J.P. Hamilton, C.R. Buell, M. Causse, S. Wijeratne, and D.M. Francis. 2012. Development of a large SNP genotyping array and generation of high-density genetic maps in tomato. PLoS ONE 7:e40563. doi:10.1371/journal.pone.0040563

SOL Genomics Network. 2014. Reference sequence. SOL Genomics Network, USA. https://solgenomics.net/jbrowse_solgeno mics/?data=data\%2Fjson\%2FSL4.0\&loc=SL4.0ch04\%3A1 ..64459972\&tracks=DNA\&highlight= $($ accessed Aug. 2014) .

Song, X., X. Sun, and T. Zhang. 2006. Segregation distortion and its effect on genetic mapping in plants. Chin. J. Agric. Biotechnol. 3(3):163-169. doi:10.1079/CJB2006110

Tovar-Méndez, A., A. Kumar, K. Kondo, A. Ashford, Y.S. Beek, L. Weich, P.A. Bedinger, and B.A. McClure. 2014. Restoring pistil-side self-incompatibility factors recapitulates an interspecific reproductive barrier between tomato species. Plant J. 77:727-736. doi:10.1111/tpj.12424

Xu. Y., ZhuL., J. Xiao, N. Huang, and S.R. McCouch. 1997. Chromosomal regions associated with segregation distortion of molecular markers in F2., backcross., doubled haploid, and recombinant inbred populations in rice (Oryza sativa L.). Mol. Gen. Genet. 253:535-545. doi:10.1007/s004380050355

Zamir, D., and Y. Tadmor. 1986. Unequal segregation of nuclear genes in plants. Bot. Gazette 147:355-358. doi:10.1086/337602 